\title{
Constructing polynomial systems with many positive solutions using tropical geometry
}

\author{
Boulos El Hilany ${ }^{1,2}$
}

Received: 4 May 2017 / Accepted: 26 December 2017 / Published online: 10 February 2018

C The Author(s) 2018. This article is an open access publication

\begin{abstract}
The number of positive solutions to a system of two polynomials in two variables defined over the field of real numbers with a total of five distinct monomials cannot exceed 15. All previously known examples have at most 5 positive solutions. The main result of this paper is the construction of a system as above having 7 positive solutions. This is achieved using tools developed in tropical geometry. When the corresponding tropical hypersurfaces intersect transversally, one can easily estimate the positive solutions to the system using the classical combinatorial patchworking for complete intersections. We apply this generalization to construct a system as above having 6 positive solutions. We also show that this bound is sharp. Consequently, our main result is proved using non-transversal intersections of tropical curves.
\end{abstract}

Keywords Real algebraic geometry · Tropical geometry · Solving polynomial systems · Fewnomial theory

Mathematics Subject Classification 14P99 $\cdot$ 14T05 $\cdot$ 13P15 $\cdot 14 \mathrm{P} 25$

\section{Introduction and statement of the main results}

The support of a system of Laurent polynomials is the set of points $w \in \mathbb{Z}^{n}$ corresponding to monomials $x^{w}=x_{1}^{w^{1}} \cdots x_{n}^{w^{n}}$ appearing in that system with non-zero coefficient. Consider a system

$凶$ Boulos El Hilany

boulos.el-hilany@uni-tuebingen.de

1 Max-Planck-Institut für Mathematik, Office B12, Vivatsgasse 7, 53111 Bonn, Germany

2 Fachbereich Mathematik, Eberhard Karls Universität Tübingen, Auf der Morgenstelle 10, 72076 Tübingen, Germany 


$$
\left\{\begin{array}{c}
f_{1}\left(x_{1}, \ldots, x_{n}\right)=0 \\
\vdots \\
f_{n}\left(x_{1}, \ldots, x_{n}\right)=0
\end{array}\right.
$$

of polynomials defined over $\mathbb{R}\left[x_{1}^{ \pm 1}, \ldots, x_{n}^{ \pm 1}\right]$ and supported on a set $\mathcal{W} \subset \mathbb{Z}^{n}$. Counting real solutions outside the coordinate hyperplane is a classical problem in algebraic geometry. However, this turns out to be a difficult task especially when dealing with polynomials of high degree or high number of monomials. Suppose a positive integer $k$ and an arbitrary system (1.1) supported on a set $\mathcal{W}$ consisting of $n+k+1$ distinct points are given. A well-justified question then arises regarding whether there exists an upper bound on the number of real solutions to the above system that depends only on the numbers $k$ and $n$. In his book [12], Khovanskii made a breakthrough concerning this question by providing an affirmative answer to it via an explicit upper bound

$$
2^{\left(\begin{array}{c}
n+k \\
2
\end{array}\right)}(n+1)^{n+k}
$$

on the number of non-degenerate positive solutions (i.e. those contained in the positive orthant of $\mathbb{R}^{n}$ ) to (1.1) (c.f. [20, Chapter 5] for more background). The above upper bound was later significantly reduced by Bihan and Sottile [6] to be

$$
\frac{e^{2}+3}{4} 2^{\left({ }_{2}^{k}\right)} n^{k} .
$$

Sharp bounds, however, on the number of positive solutions are already known only in some special cases. For example, Descartes' rule of sign provides a sharp upper bound $k+1$ on the number of positive solutions (counted with multiplicities) to a univariate real polynomial having $k+2$ distinct monomials. Also, Bihan proved in [2] that if $k=1$, then $n+1$ is a sharp upper bound on the number of positive solutions to (1.1).

This paper concerns one of the first cases where the sharp upper bound on the number of non-degenerate positive solutions to (1.1) is still unknown. Namely, with respect to the above notations, our main result involves a system (1.1) satisfying $n=k=2$.

Theorem 1.1 There exists a real system (1.1) of two polynomials in two variables supported on a set $\mathcal{W}$ of five distinct points in $\mathbb{Z}^{2}$ and having seven non-degenerate positive solutions.

It was proven in [6] that a sharp bound to such a system (of type $n=k=2$ for short) is not greater than 15 . On the other hand, the best examples had only 5 non-degenerate positive solutions. The first such published system, made by Haas [9], is a construction of two real bivariate trinomials, after which other similar examples of systems having 5 positive solutions followed in [7]. The authors in the latter paper also showed that such systems (consisting of two real bivariate trinomials) are rare in the following sense. They proved that if we pick a point at random in the discriminant variety of coefficient spaces of polynomial systems composed of two bivariate trinomials with fixed exponent vectors, this point has probability of order $10^{-9}$ to be located in 
a chamber (connected component of the complement) containing systems with the maximal number five of positive solutions.

In this paper, we consider real systems of type $n=k=2$ in their full generality (i.e. not only the case of two trinomials). The motivation behind this work is to adopt some of the tools developed in tropical geometry in order to construct real polynomial systems of type $n=k=2$ that give more than five positive solutions. Tropical geometry is a new domain in mathematics that is situated at the junction of fields such as toric geometry, complex or real geometry, and combinatorics [14-16]. It turns out that Sturmfels' Theorem [22] can be reformulated in the context of tropical geometry (see [25]). This makes the latter an effective tool to construct polynomial systems with prescribed support and many positive solutions. The principal idea is to consider a family of polynomial systems

$$
\left\{\begin{array}{l}
P_{1, t}(x, y)=0 \\
P_{2, t}(x, y)=0
\end{array}\right.
$$

of type $n=k=2$ with special 1-parametrized coefficients $a_{i}^{j}(t)$ for $(i, j) \in$ $\{1,2\} \times\{1, \ldots, 5\}$ (we will define this parametrisation in Sect. 2). We then associate to $P_{1, t}$ and $P_{2, t}$ tropical curves $T_{1}, T_{2} \subset \mathbb{R}^{2}$ (see Sect. 2.1), which can be viewed as piecewise-linear combinatorial objects that keep track of much of the information about the (parametrized) solutions to (1.4). Assume first that the associated tropical curves intersect transversally in a finite set of points $\mathcal{S}$ (i.e. the cardinality of $\mathcal{S}$ does not change after perturbations). Then, Sturmfels' generalization of Viro's Theorem (see Theorem 3.2) states that there exists a bijection between the set of positive solutions to the real system obtained from (1.4) by taking $t>0$ small enough, and the subset $\mathcal{S}_{+} \subseteq \mathcal{S}$ of common points in the positive facets of $T_{1}$ and of $T_{2}$ (see Definition 3.1). Therefore, similarly to the famous Viro's patchworking technique (see Theorem 3.1), this approach provides a combinatorial method for construction in real algebraic geometry. If the system (1.4) is of type $n=k=2$, then the number of transversal intersection points of $T_{1}$ and $T_{2}$ is bounded from above by six (see Lemma 3.4). It was previously unknown whether this upper bound can be attained. We prove that this bound is sharp and can be realized using only the above set $\mathcal{S}_{+}$.

Proposition 1.2 There exist two plane tropical curves $T_{1}$ and $T_{2}$ defined by equations containing a total of five monomials and which have six transversal intersection points. Moreover, each such point belongs to a positive facet of $T_{1}$ and a positive facet of $T_{2}$.

Due to Theorem 3.2, the construction made for proving the latter result also gives a construction of a real polynomial system of type $n=k=2$ that has six positive solutions. Furthermore it is clear from Theorem 3.3 and Lemma 3.4 that one cannot hope to improve the result in Proposition 1.2 when restricting to polynomial systems of type $n=k=2$ with tropical curves intersecting transversally. Consequently, in order to obtain a better construction, we consider real parametrized polynomial systems (1.4) of type $n=k=2$, having only non-degenerate solutions, and whose tropical curves $T_{1}$ and $T_{2}$ intersect in a non-empty set that does not consist entirely of transversal points. 
A consequence of an important result due to Kapranov [10] is that the set $T_{1} \cap T_{2}$ contains the image under the valuation map (see Sect. 2.1) of the solutions to (1.4). The strategy for proving Theorem 1.1 is to deduce, from the combinatorial types of the connected components of $T_{1} \cap T_{2}$, the number of non-degenerate positive solutions to (1.4) that map to $T_{1} \cap T_{2}$. Namely, we decompose those connected components to linear pieces $\xi$ which can either have dimension zero or one. Then, to each such $\xi$, we associate a certain polynomial sub-system of (1.4), called reduced real systems with respect to $\xi$ (see [8, Chapter 2.2.6]). All together, those reduced systems approximate all the non-degenerate parametrized solutions to (1.4) (see $[11,17,18]$ and [5]). We adapt this approach to our setting by considering a particular type of parametrized non-degenerate solutions $\left(\alpha_{1}(t), \alpha_{2}(t)\right)$, which we also call positive (i.e. the firstorder terms of $\alpha_{1}(t)$ and $\alpha_{2}(t)$ have positive coefficients). This refinement constitutes an important benefit concerning our construction. Specifically, when $t>0$ is small enough, the system (1.4) produces a real system of type $n=k=2$ having a set of non-degenerate positive solutions that is in a bijective correspondence with the set of the non-degenerate positive solutions to (1.4).

In [8, Chapter 6.4], a case-by-case analysis was made, for a family of systems (1.4) of type $n=k=2$, to identify the few classes of candidates of such systems that have more than six positive solutions. The construction described in the present paper is based on one such candidate.

This paper is organized as follows. We introduce in Sect. 2 some basic notions of tropical geometry. In Sect. 3, we give a description of the tropical reformulation of Viro's Patchworking Theorem and its generalization followed by the proof of Proposition 1.2. Finally, Sect. 4 is devoted to the proof of Theorem 1.1.

\section{A brief introduction to tropical geometry}

We state in this section some of the well-known facts about tropical geometry (see [4], and the references therein), much of the exposition and notations in this section are taken from $[1,5,19]$.

Definition 2.1 A polyhedral subdivision of a convex polytope $\Delta \subset \mathbb{R}^{n}$ is a set of convex polytopes $\left\{\Delta_{i}\right\}_{i \in I}$ such that

- $\cup_{i \in I} \Delta_{i}=\Delta$, and

- if $i, j \in I$, then if the intersection $\Delta_{i} \cap \Delta_{j}$ is non-empty, it is a common face of the polytope $\Delta_{i}$ and the polytope $\Delta_{j}$.

Definition 2.2 Let $\Delta$ be a convex polytope in $\mathbb{R}^{n}$ and let $\tau$ denote a polyhedral subdivision of $\Delta$ consisting of convex polytopes. We say that $\tau$ is regular if there exists a continuous, convex, piecewise-linear function $\varphi: \Delta \rightarrow \mathbb{R}$ such that the polytopes of $\tau$ are exactly the domains of linearity of $\varphi$.

Let $\Delta$ be an integer convex polytope in $\mathbb{R}^{n}$ and let $\phi: \Delta \cap \mathbb{Z}^{n} \rightarrow \mathbb{R}$ be a function. We denote by $\hat{\Delta}(\phi)$ the convex hull of the graph of $\phi$, i.e.,

$$
\hat{\Delta}(\phi):=\operatorname{Conv}\left(\left\{(i, \phi(i)) \in \mathbb{R}^{n+1} \mid i \in \Delta \cap \mathbb{Z}^{n}\right\}\right) .
$$


Then the polyhedral subdivision of $\Delta$ induced by projecting the union of the lower faces of $\hat{\Delta}(\phi)$ onto the first $n$ coordinates, is regular.

\subsection{Tropical polynomials and hypersurfaces}

A locally convergent generalized Puiseux series is a formal series of the form

$$
a(t)=\sum_{r \in R} \alpha_{r} t^{r}
$$

where $R \subset \mathbb{R}$ is a well-ordered set, all $\alpha_{r} \in \mathbb{C}$, and the series is convergent for $t>0$ small enough. We denote by $\mathbb{K}$ the set of all locally convergent generalized Puiseux series. It is clearly a field of characteristic 0 , which turns out to be algebraically closed. The field $\mathbb{K}$ can be equipped with a non-archimedian valuation defined as follows:

$$
\begin{aligned}
& \text { val : } \mathbb{K} \quad \longrightarrow \quad \mathbb{R} \cup\{-\infty\}
\end{aligned}
$$

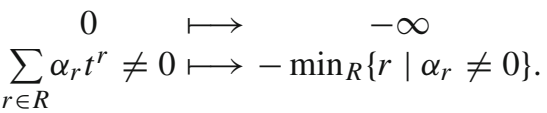

Extend the valuation to a map Val : $\mathbb{K}^{n} \rightarrow(\mathbb{R} \cup\{-\infty\})^{n}$ by evaluating val coordinatewise, i.e. $\operatorname{Val}\left(z_{1}, \ldots, z_{n}\right)=\left(\operatorname{val}\left(z_{1}\right), \ldots, \operatorname{val}\left(z_{n}\right)\right)$.

Notation 2.1 Let coef $(a(t))$ denote the coefficient $\alpha_{-\operatorname{val}(a(t))}$ of the first term of $a(t)$ following the increasing order of the exponents of $t$. We extend coef to a map Coef $: \mathbb{K}^{n} \rightarrow \mathbb{R}^{n}$ by taking coef coordinate-wise, i.e. $\operatorname{Coef}\left(a_{1}(t), \ldots, a_{n}(t)\right)=$ $\left(\operatorname{coef}\left(a_{1}(t)\right), \ldots, \operatorname{coef}\left(a_{n}(t)\right)\right)$.

We use the convention that for any $s \in \mathbb{K}$, we have $\operatorname{coef}(s)=0 \Leftrightarrow s=0$ and $\operatorname{val}(s)=-\infty \Leftrightarrow s=0$.

An element $a(t)=\sum_{r \in R} \alpha_{r} t^{r}$ of $\mathbb{K}$ is said to be real if $\alpha_{r} \in \mathbb{R}$ for all $r$, and positive if $a(t)$ is real and coef $(a(t))>0$. Denote by $\mathbb{R} \mathbb{K}$ (resp. $\mathbb{R} \mathbb{K}_{>0}$ ) the subfield of $\mathbb{K}$ composed of real (resp. positive) series. Since elements of $\mathbb{K}$ are convergent for $t>0$ small enough, an algebraic variety over $\mathbb{K}($ resp. $\mathbb{R} \mathbb{K})$ can be seen as a one-parametric family of algebraic varieties over $\mathbb{C}$ (resp. $\mathbb{R}$ ).

Consider a polynomial

$$
f(z):=\sum_{w \in \mathcal{W}} c_{w} z^{w} \in \mathbb{K}\left[z_{1}^{ \pm 1}, \ldots, z_{n}^{ \pm 1}\right]
$$

with $\mathcal{W}$ a finite subset of $\mathbb{Z}^{n}$ and all $c_{w}$ are non-zero. Let $V_{f}=\left\{z \in\left(\mathbb{K}^{*}\right)^{2} \mid f(z)=0\right\}$ be the zero set of $f$ in $\left(\mathbb{K}^{*}\right)^{n}$. The tropical hypersurface $V_{f}^{\text {trop }}$ associated to $f$ is the image under Val of $V_{f}$ :

$$
V_{f}^{\text {trop }}=\operatorname{Val}\left(V_{f}\right) \subset \mathbb{R}^{n}
$$


Now, define

$$
\begin{aligned}
v_{f}: \mathbb{Z}^{n} \longrightarrow & \mathbb{R} \cup\{-\infty\} \\
w & \longmapsto \begin{cases}-\operatorname{val}\left(c_{w}\right), & \text { if } w \in \mathcal{W}, \\
-\infty, & \text { otherwise }\end{cases}
\end{aligned}
$$

Its Legendre transform is a piecewise-linear convex function

$$
\begin{aligned}
\mathcal{L}\left(v_{f}\right): \mathbb{R}^{n} & \longrightarrow \\
x & \longmapsto \max _{w \in \mathcal{W}}\left\{\langle x, w\rangle-v_{f}(w)\right\},
\end{aligned}
$$

where $\langle\rangle:, \mathbb{R}^{n} \times \mathbb{R}^{n} \rightarrow \mathbb{R}$ is the standard eucledian product. The set of points $x \in \mathbb{R}^{n}$ at which $\mathcal{L}\left(v_{f}\right)$ is not differentiable is called the corner locus of $\mathcal{L}\left(v_{f}\right)$. We have the fundamental Theorem of Kapranov [10].

Theorem 2.2 (Kapranov) A tropical hypersurface $V_{f}^{\text {trop }}$ of a polynomial $f$ defined over an algebraically closed field is the corner locus of the Legendre transform $\mathcal{L}\left(v_{f}\right)$.

For a positive integer $l$, we define the tropical summation of $x_{1}, \ldots, x_{l} \in$ $\mathbb{R} \cup\{-\infty\}$ as their usual maximum $\max \left(x_{1}, \ldots, x_{l}\right)$, and the tropical multiplication as their usual sum $\sum_{i=1}^{l} x_{i}$. A tropical polynomial is a polynomial in $\mathbb{R}\left[x_{1}, \ldots, x_{n}\right]$, where the addition and multiplication are the tropical ones. Hence, a tropical polynomial is given by a maximum of finitely many affine functions whose linear parts have integer coefficients and constant parts are real numbers.

The tropicalization of a polynomial $f$ as above is the tropical polynomial

$$
f_{\text {trop }}(x)=\max _{w \in \mathcal{W}}\left\{\langle x, w\rangle+\operatorname{val}\left(c_{w}\right)\right\}
$$

Note that the latter coincides with the piecewise-linear convex function $\mathcal{L}\left(v_{f}\right)$ defined above. Therefore, Theorem 2.2 asserts that $V_{f}^{\text {trop }}$ is the corner locus of $f_{\text {trop }}$. Conversely, the corner locus of any tropical polynomial is a tropical hypersurface.

Example 2.3 A polynomial $f \in \mathbb{R} \mathbb{K}\left[z_{1}, z_{2}\right]$ with equation

$$
f\left(z_{1}, z_{2}\right)=-t+z_{1}-t z_{1}^{2}-z_{1} z_{2}+z_{2}+t z_{2}^{2}
$$

its associated tropical polynomial is

$$
f_{\text {trop }}\left(x_{1}, x_{2}\right)=\max \left\{-1, x_{1}, 2 x_{1}-1, x_{1}+x_{2}, x_{2}, 2 x_{2}-1\right\}
$$

and the corresponding tropical hypersurface is shown in Fig. 1 on the left. 

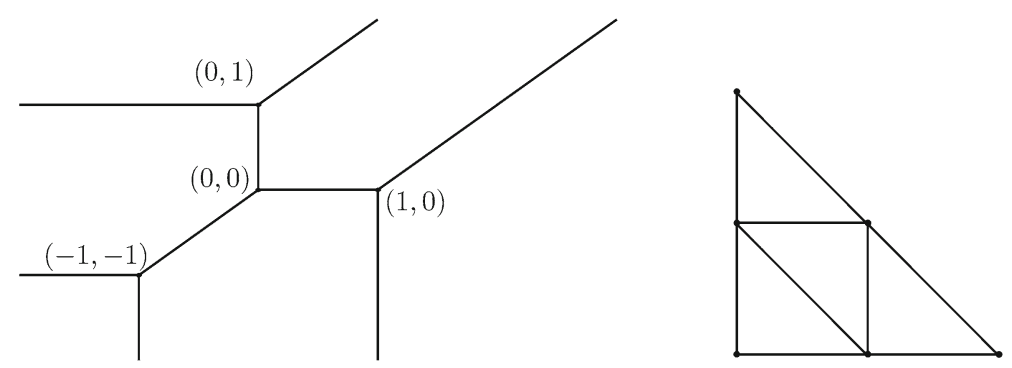

Fig. 1 An example of a tropical conic in $\mathbb{R}^{2}$, and its dual subdivision

\subsection{Tropical hypersurfaces and subdivisions}

Keeping with the same notation as above, the tropical hypersurface $V_{f}^{\text {trop }}$ is an $(n-1)$ dimensional piecewise-linear complex which produces a polyhedral subdivision $\Xi$ of $\mathbb{R}^{n}$. This subdivision induces a regular subdivision $\tau$ of the Newton polytope $\Delta(f)$ of $f$ in the following way (see [1, Section 3] for more details). The elements of $\Xi$, called cells, have rational slopes. The $n$-dimensional cells of $\Xi$ are the closures of the connected components of the complement of $V_{f}^{\text {trop }}$ in $\mathbb{R}^{n}$. The lower dimensional cells of $\Xi$ are contained in $V_{f}^{\text {trop }}$ and we will just say that they are cells of $V_{f}^{\text {trop }}$.

Given a cell $\xi$ of $V_{f}^{\text {trop }}$ and a point $x$ in the relative interior of $\xi$, the set

$$
\mathcal{I}_{\xi}=\left\{w \in \mathcal{W} \mid f_{\text {trop }}(x)=\langle x, w\rangle+\operatorname{val}\left(c_{w}\right)\right\}
$$

does not depend on $x$. All together the polyhedra $\Delta_{\xi}:=\operatorname{Conv}\left(\mathcal{I}_{\xi}\right)$ form a subdivision $\tau$ of $\Delta(f)$ called the dual subdivision, and the polyhedron $\Delta_{\xi}$ is called the dual of $\xi$.

Consider now polynomials $f_{1}, \ldots, f_{r} \in \mathbb{K}\left[z_{1}^{ \pm 1}, \ldots, z_{n}^{ \pm 1}\right]$. For $i=1, \ldots, r$, let $\mathcal{W}_{i} \subset \mathbb{Z}^{n}$ (resp. $\Delta_{i} \subset \mathbb{R}^{n}, T_{i} \subset \mathbb{R}^{n}$ ) denote the support (resp. Newton polytope, tropical hypersurface) associated to $f_{i}$.

The union of these tropical hypersurfaces defines a piecewise-linear polyhedral subdivision $\Xi$ of $\mathbb{R}^{n}$. Any non-empty cell of $\Xi$ can be written as

$$
\xi=\xi_{1} \cap \cdots \cap \xi_{r}
$$

with $\xi_{i} \in \Xi_{i}$ for $i=1, \ldots, r$, where $\Xi_{i}$ is the polyhedral subdivision of $\mathbb{R}^{n}$ produced by $T_{i}$. We require that $\xi$ does not lie in the boundary of any $\xi_{i}$, thus any cell $\xi \in \Xi$ can be uniquely written in this way. Denote by $\tau$ the mixed subdivision of the Minkowskii sum $\Delta=\Delta_{1}+\cdots+\Delta_{r}$ induced by the tropical polynomials $f_{1}, \ldots, f_{r}$. Recall that any polytope $\sigma \in \tau$ comes with a privileged representation $\sigma=\sigma_{1}+\cdots+\sigma_{r}$ with $\sigma_{i} \in \tau_{i}$ for $i=1, \ldots, r$, where each $\tau_{i}$ is the dual subdivision of $\Delta_{i}$. The above duality-correspondence applied to the (tropical) product of the tropical polynomials gives rise to the following well-known fact (see [1, Section 4] for instance). 
Proposition 2.4 There is a one-to-one duality correspondence between $\Xi$ and $\tau$, which reverses the inclusion relations, and such that if $\sigma \in \tau$ corresponds to $\xi \in \Xi$, then

(1) if $\xi=\xi_{1} \cap \cdots \cap \xi_{r}$ with $\xi_{i} \in \Xi_{i}$ for $i=1, \ldots, r$, then $\sigma$ has representation $\sigma=\sigma_{1}+\cdots+\sigma_{r}$ where each $\sigma_{i}$ is the polytope dual to $\xi_{i}$.

(2) $\operatorname{dim} \xi+\operatorname{dim} \sigma=n$,

(3) the cell $\xi$ and the polytope $\sigma$ span orthonogonal real affine spaces,

(4) the cell $\xi$ is unbounded if and only if $\sigma$ lies on a proper face of $\Delta$.

Definition 2.3 A cell $\xi$ is transversal if it satisfies $\operatorname{dim}\left(\Delta_{\xi}\right)=\operatorname{dim}\left(\Delta_{\xi_{1}}\right)+\cdots+$ $\operatorname{dim}\left(\Delta_{\xi_{r}}\right)$, and it is non transversal if the previous equality does not hold.

\section{First construction: transversal case}

\subsection{Generalized Viro theorem and tropical reformulation}

Viro's combinatorial patchworking $[23,24]$ can be interpreted via the so-called "dequantization" method (see [25]). In what follows, we use tropical hypersurfaces to formulate this description.

Let $\mathcal{W} \subset \mathbb{Z}^{n}$ be a finite set of lattice points, and denote by $\Delta$ the convex hull of $\mathcal{W}$. Assume that $\operatorname{dim} \Delta=n$ and let $\varphi: \Delta \rightarrow \mathbb{Z}$ be a function inducing a regular triangulation $\tau_{\varphi}$ of the integer convex polytope $\Delta$ (see Definition 2.2), such that $\mathcal{W}$ is the set of vertices of this triangulation. For each $w \in \mathcal{W}$, fix a non-zero real number $c_{w}$. For each positive real number $t$, we consider a Laurent polynomial

$$
f_{t}\left(z_{1}, \ldots, z_{n}\right)=\sum_{w \in \mathcal{W}} c_{w} t^{\varphi(w)} z^{w} .
$$

We consider $g:=f_{t}$ as a polynomial defined over the field of real generalized locally convergent Puiseux series, where each coefficient $c_{w} t^{\varphi(w)} \in \mathbb{R} \mathbb{K}^{*}$ of $g$ has only one term. Therefore $\operatorname{coef}\left(c_{w} t^{\varphi(w)}\right)=c_{w}, \operatorname{val}\left(c_{w} t^{\varphi(w)}\right)=-\varphi(w)$, and we associate to $g$ a tropical hypersurface $V_{g}^{\text {trop }}$ as defined in Sect. 2.1. Recall that $V_{g}^{\text {trop }}$ induces a subdivision $\Xi_{g}$ of $\mathbb{R}^{n}$ that is dual to $\tau_{\varphi}$. We define for each $n$-cell $\xi \in \Xi_{g}$, dual to a 0 -face (vertex) $w$ of the triangulation $\tau_{\varphi}$, a sign $\epsilon(w) \in\{+,-\}$, to be equal to the sign of $c_{w}$.

Definition 3.1 The positive part, denoted by $V_{g,+}^{\text {trop }}$, is the subcomplex of $V_{g}^{\text {trop }}$ consisting of all $(n-1)$-cells of $V_{g}^{\text {trop }}$ that are adjacent to two $n$-cells of $\Xi_{g}$ having different signs (see the left part of Fig. 2 for an example). A positive facet $\xi_{+}$is an $(n-1)$-dimensional cell of $V_{g,+}^{\text {trop }}$.

Theorem 3.1 (Viro) Let $f_{t} \in \mathbb{R} \mathbb{K}\left[z_{1}^{ \pm 1}, \ldots, z_{n}^{ \pm 1}\right]$ be a polynomial (3.1), denote by $V_{+}\left(f_{t}\right) \subset\left(\mathbb{R}_{>0}\right)^{n}$ the intersection of the zero set of $f_{t}$ with the positive orthant of $\mathbb{R}^{n}$, and by $V_{g,+}^{\text {trop }} \subset \mathbb{R}^{n}$ the positive part of the tropical hypersurface corresponding to $g:=f_{t}$. Then for sufficiently small $t>0$, there exists a homeomorphism $\left(\mathbb{R}_{>0}\right)^{n} \rightarrow$ $\mathbb{R}^{n}$ sending $V_{+}\left(f_{t}\right) \subset\left(\mathbb{R}_{>0}\right)^{n}$ to $V_{g,+}^{\text {trop }} \subset \mathbb{R}^{n}$. 

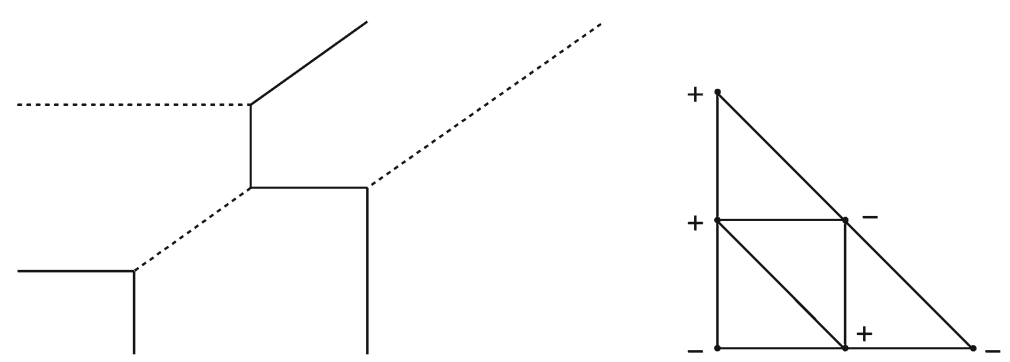

Fig. 2 The positive part of the tropical hypersurface associated to $-t+z_{1}-t z_{1}^{2}-z_{1} z_{2}+z_{2}+t z_{2}^{2}$ is represented as the union of the solid segments and solid half-rays

In fact, $\mathrm{O}$. Viro proves a more general version of Theorem 3.1 by similarly describing the whole zero set $V\left(f_{t}\right) \subset \mathbb{R}^{n}$ (not only $V\left(f_{t}\right) \cap\left(\mathbb{R}_{>0}\right)^{n}$ ). Later, B. Sturmfels generalized Viro's method for complete intersections in [22]. We give now a tropical reformulation of one of the main Theorems of [22].

Theorem 3.2 (Sturmfels) Let $f_{1, t}, \ldots, f_{r, t} \in \mathbb{R} \mathbb{K}\left[z_{1}^{ \pm 1}, \ldots, z_{n}^{ \pm 1}\right]$ be $r$ polynomials of the form (3.1), and denote by $V_{+}\left(f_{1, t}, \ldots, f_{r, t}\right)$ their common real zero-locus in the positive orthant of $\mathbb{R}^{n}$. For $i=1, \ldots, r$, let $V_{g_{i},+}^{\text {trop }}$ denote the positive part of the tropical hypersurface $V_{g_{i}}^{\text {trop }} \subset \mathbb{R}^{n}$ corresponding to $g_{i}:=f_{i, t}$, and assume that $V_{g_{1}}^{\text {trop }} \cap \cdots \cap V_{g_{r}}^{\text {trop }}$ is a union of transversal cells (see Definition 2.3). Then for sufficiently small $t>0$, there exists a homeomorphism $\left(\mathbb{R}_{>0}\right)^{n} \rightarrow \mathbb{R}^{n}$ sending the real algebraic set $V_{+}\left(f_{1, t}, \ldots, f_{r, t}\right) \subset\left(\mathbb{R}_{>0}\right)^{n}$ to the intersection $V_{g_{1},+}^{\text {trop }} \cap \cdots \cap V_{g_{r},+}^{\text {trop }} \subset \mathbb{R}^{n}$.

Similarly to Viro's work, B. Sturmfels generalizes Theorem 3.2 for the zero set $V\left(f_{1, t}, \ldots, f_{r, t}\right) \subset \mathbb{R}^{n}$ (see [22, Theorem 5]).

\subsection{Tropical transversal intersection points for bivariate polynomials}

Consider a system

$$
\left\{\begin{array}{l}
f_{1, t}\left(z_{1}, z_{2}\right)=0, \\
f_{2, t}\left(z_{1}, z_{2}\right)=0,
\end{array}\right.
$$

where $f_{1, t}, f_{2, t}$ are polynomials of the form (3.1). For the rest of this section, we assume that the intersection of the tropical curves $V_{g_{1}}^{\text {trop }}, V_{g_{2}}^{\text {trop }} \subset \mathbb{R}^{2}$, corresponding to $g_{1}:=f_{1, t}$ and $g_{2}:=f_{2, t}$ respectively, consists of a union of transversal cells. This implies that the positive parts $V_{g_{1},+}^{\text {trop }}$ and $V_{g_{2},+}^{\text {trop }}$ of $V_{g_{1}}^{\text {trop }}$ and $V_{g_{2}}^{\text {trop }}$ respectively, is a finite (possibly empty) set of points in $\mathbb{R}^{2}$. Each point of $V_{g_{1},+}^{\text {trop }} \cap V_{g_{2},+}^{\text {trop }}$ is expressed in a unique way as a transversal intersection $\xi_{1,+} \cap \xi_{2,+}$, where for $i=1,2$, the cell $\xi_{i,+} \subset V_{g_{i},+}^{\text {trop }}$ is a positive cell (see Definition 3.1). In this section, we use Theorem 3.2 to prove Proposition 1.2.

Bihan [3] gave an upper bound on $\left|V_{g_{1}}^{\text {trop }} \cap V_{g_{2}}^{\text {trop }}\right|$ (and thus on $\left|V_{g_{1},+}^{\text {trop }} \cap V_{g_{2},+}^{\text {trop }}\right|$ ) corresponding to a bivariate system (3.2) in two equations. Namely, given two finite sets $\mathcal{W}_{1}$ and $\mathcal{W}_{2}$ in $\mathbb{R}^{2}$, and for any non-empty $I \subset\{1,2\}$, write $\mathcal{W}_{I}$ for the set of 
points $\sum_{i \in I} w_{i}$ over all $w_{i} \in \mathcal{W}_{i}$ with $i \in I$. The associated discrete mixed volume of $\mathcal{W}_{1}$ and $\mathcal{W}_{2}$ is defined as

$$
D\left(\mathcal{W}_{1}, \mathcal{W}_{2}\right)=\sum_{I \subset[r]}(-1)^{r-|I|}\left|\mathcal{W}_{I}\right|,
$$

where the sum is taken over all subsets $I$ of $\{1,2\}$ including the empty set with the convention that $\left|\mathcal{W}_{\emptyset}\right|=1$. Denote by $\mathcal{W}_{i}$ the support of $g_{i}$ for $i=1,2$.

Theorem 3.3 (Bihan) If the intersection locus $V_{g_{1}}^{\text {trop }} \cap V_{g_{2}}^{\text {trop }}$ of the tropical curves corresponding to the polynomials in (3.2) consists only of transversal points, then $\left|V_{g_{1}}^{\text {trop }} \cap V_{g_{2}}^{\text {trop }}\right|$ is less or equal to the discrete mixed volume $D\left(\mathcal{W}_{1}, \mathcal{W}_{2}\right)$ of the corresponding supports.

When $\left|\mathcal{W}_{1} \cup \mathcal{W}_{2}\right|=4$, then the bound of Theorem 3.3 is 3 , and is sharp (see [2]). However, we do not know if the discrete mixed volume bound is sharp for any polynomial system with 2 equations in 2 variables satisfying that the associated tropical curves intersect transversally.

\subsection{Restriction to the case $n=k=2$}

We need the following result.

Lemma 3.4 If $\mathcal{W}_{1}$ and $\mathcal{W}_{2}$ are two subsets of $\mathbb{Z}^{2}$ satisfying $\left|\mathcal{W}_{1} \cup \mathcal{W}_{2}\right|=5$, then the discrete mixed volume $D\left(\mathcal{W}_{1}, \mathcal{W}_{2}\right)$ does not exceed six.

Proof Recall that $\left|\mathcal{W}_{1} \cup \mathcal{W}_{2}\right|=5$. We distinguish the five possible cases $\left|\mathcal{W}_{1} \cap \mathcal{W}_{2}\right|=i$ for $i=1, \ldots, 5$, and prove the result only for $i=3,4$ since the case $i=5$ is proven in [3] and the remaining cases are similar. The discrete mixed volume of $\mathcal{W}_{1}$ and $\mathcal{W}_{2}$ is expressed as

$$
D\left(\mathcal{W}_{1}, \mathcal{W}_{2}\right)=\left|\mathcal{W}_{1}+\mathcal{W}_{2}\right|-\left|\mathcal{W}_{1}\right|-\left|\mathcal{W}_{2}\right|+1
$$

Note that, since $\mathcal{W}_{1} \cap \mathcal{W}_{2} \neq \emptyset$, one can write

$$
\left|\mathcal{W}_{1}+\mathcal{W}_{2}\right| \leq\left|\mathcal{W}_{1}\right| \cdot\left|\mathcal{W}_{2}\right|-\left(\begin{array}{c}
\left|\mathcal{W}_{1} \cap \mathcal{W}_{2}\right| \\
2
\end{array}\right)
$$

If $\left|\mathcal{W}_{1} \cap \mathcal{W}_{2}\right|=4$, then the cardinality of one of the two sets, say $\mathcal{W}_{1}$, is equal to four, which in turn means that $\left|\mathcal{W}_{2}\right|=5$. Using (3.5), we compute that $\left|\mathcal{W}_{1}+\mathcal{W}_{2}\right| \leq 14$, and thus (3.4) gives $D\left(\mathcal{W}_{1}, \mathcal{W}_{2}\right) \leq 6$.

Assume now that $\left|\mathcal{W}_{1} \cap \mathcal{W}_{2}\right|=3$. If $\left|\mathcal{W}_{1}\right|=3$ and $\left|\mathcal{W}_{2}\right|=5$ (the case where $\left|\mathcal{W}_{1}\right|=5$ and $\left|\mathcal{W}_{2}\right|=3$ is treated similarly), then (3.5) gives $\left|\mathcal{W}_{1}+\mathcal{W}_{2}\right| \leq 12$, and thus we get $D\left(\mathcal{W}_{1}, \mathcal{W}_{2}\right) \leq 5$. Finally, if $\left|\mathcal{W}_{1}\right|=\left|\mathcal{W}_{2}\right|=4$, then $\left|\mathcal{W}_{1}+\mathcal{W}_{2}\right| \leq 13$, from which we deduce that $D\left(\mathcal{W}_{1}, \mathcal{W}_{2}\right) \leq 6$, and the statement is proved.

We finish this section by proving Proposition 1.2.

Proof of Proposition 1.2 Figure 3 shows that the tropical curves associated to the equations of the system 

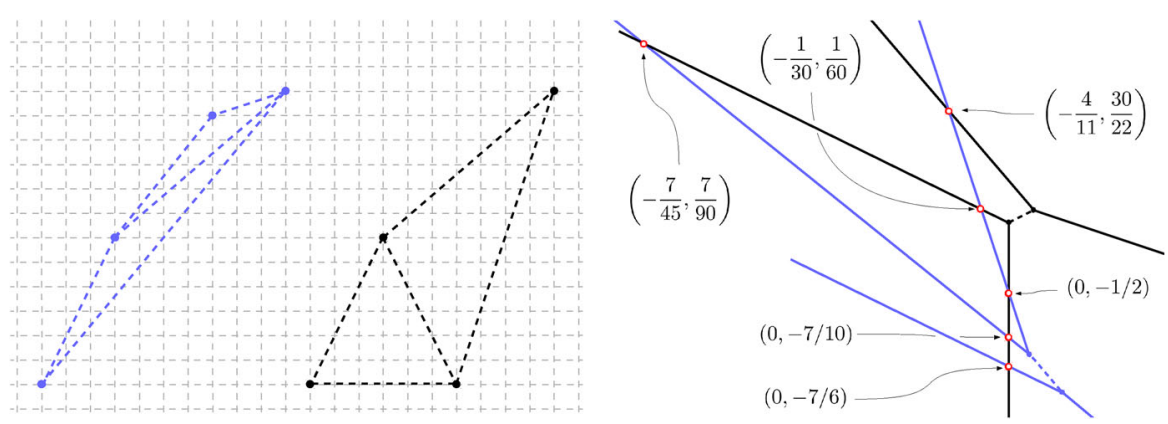

Fig. 3 To the left: the Newton polytopes and subdivisions associated to the equations of (3.6). To the right: the corresponding tropical curves intersecting at 6 transversal points

$$
\left\{\begin{aligned}
-1+t^{12}+x^{6}+x^{3} y^{6}-t x^{10} y^{12} & =0 \\
-t^{12}+t^{5} x^{3} y^{6}-t^{1.5} x^{7} y^{11}+t x^{10} y^{12} & =0
\end{aligned}\right.
$$

intersect at six transversal intersection points.

As explained before, Theorem 3.2 shows that for a positive $t$ small enough, the system (3.6) becomes a real bivariate polynomial system of type $n=k=2$ having 6 positive solutions.

\section{Second construction: non-transversal case}

Following the notation of Sect. 2.2 for the case of two tropical curves $T_{1}$ and $T_{2}$ in $\mathbb{R}^{2}$, we classify the types of cells $\xi$ of $T_{1} \cap T_{2}$ at which the two tropical curves intersect non-transversally. Any intersection locus can be decomposed into linear pieces, each of which falls down into one of three types. Any such linear piece $\xi$ can be written as an intersection $\xi_{1} \cap \xi_{2}$, where $\xi_{1}$ and $\xi_{2}$ are cells of $T_{1}$ and $T_{2}$ respectively. Assume that it is a non-transversal linear piece, we distinguish three types for such $\xi$ (Fig. 4).

- A cell $\xi$ is of type (I) if $\operatorname{dim} \xi=\operatorname{dim} \xi_{1}=\operatorname{dim} \xi_{2}=1$.

- A cell $\xi$ is of type (II) if one of the cells $\xi_{1}$, or $\xi_{2}$ is a vertex, and the other cell is an edge.

- A cell $\xi$ is of type (III) if $\xi_{1}$ and $\xi_{2}$ are vertices of the corresponding tropical curves.

\subsection{Construction}

Consider a system

$$
\left\{\begin{array}{l}
a_{0}+z_{1}^{m_{1}}+a_{2} z_{1}^{m_{2}} z_{2}^{n_{2}}+a_{3} t^{\alpha} z_{1}^{m_{3}} z_{2}^{n_{3}}=0 \\
b_{0}+z_{1}^{m_{1}}+b_{2} z_{1}^{m_{2}} z_{2}^{n_{2}}+b_{4} t^{\beta} z_{1}^{m_{4}} z_{2}^{n_{4}}=0
\end{array}\right.
$$

satisfying the following, 

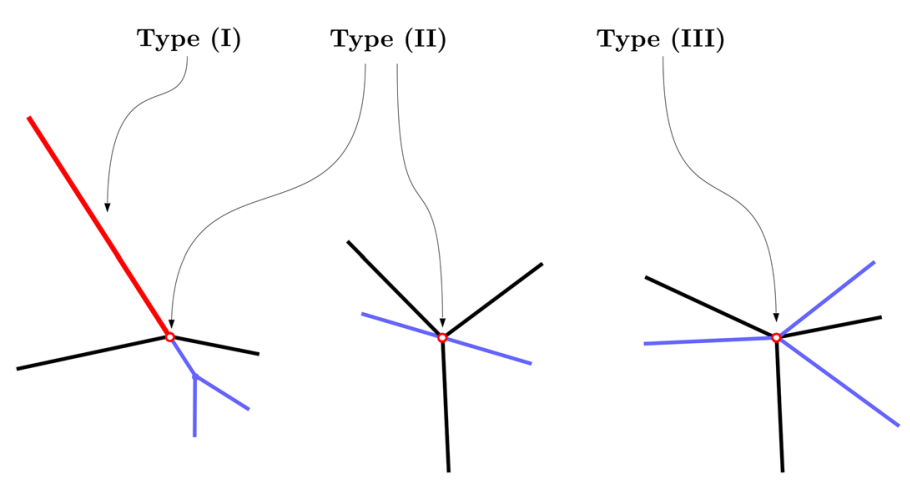

Fig. 4 The three types of non-transversal intersection cells

- all $a_{i}, b_{j}$ are real generalized Puiseux series in $t$ and having valuation zero,

- the support of (4.1) is a subset of $\mathbb{Z}^{2}$ with no three points belonging to a line, and

- the integers $m_{1}, n_{2}$ are positive and the numbers $\alpha, \beta$ are real.

This is a system of type $n=k=2$ with polynomials in the field of real generalized Puiseux series. In [8, Lemma 6.14], it is proven that to any real system of type $n=$ $k=2$ verifying some non-degeneracy conditions, one can associate a system (4.1) such that it satisfies the latter three conditions and preserves the number of nondegenerate positive solutions. We have chosen to start our construction with systems of the form (4.1) since, in terms of number of parameters, they are more manageable than generic ones of type $n=k=2$. Denote by $T_{1}$ (resp. $T_{2}$ ) the tropical curve associated to the first (resp. second equation) of (4.1). In what follows, we explain how to choose the coefficients and exponents of (4.1) such that the latter system has seven positive solutions.

First, Theorem 1.16 of [8] implies that if $\alpha \neq \beta$ or $\alpha=\beta<0$, then (4.1) has at most six positive solutions. This was done in [8, Chapter $6.6-6.7]$ via a case-by-case analysis of the coefficients and exponent vectors of the system (4.1). Namely, for each realizable intersection locus $T_{1} \cap T_{2}$, one can provide an upper bound $N(\xi)$ on the number of positive solutions to (4.1) with valuation in any linear piece $\xi$ of $T_{1} \cap T_{2}$. Then, proving that not all $N(\xi)$ can attain their maximal possible value at once, gives the upper bound six if either $\alpha \neq \beta$ or $\alpha=\beta<0$.

On the other hand, the special case where $\alpha=\beta=0$ was not treated in [8], for the latter approach does not provide an upper bound better than fifteen, which is previously-known (see [6]) and remains unclear whether it is sharp or not.

We assume henceforth that $\alpha=\beta>0$. From the equations appearing in (4.1), it is easy to deduce that, since $\alpha, \beta>0$, the intersection locus of tropical curves $T_{1}$ and $T_{2}$ contains a non-transversal intersection point of type (III) at the origin $v_{0}=(0,0)$. The 3-valent vertex $v_{0}$ is adjacent to three 1-dimentional linear pieces of $T_{1} \cap T_{2}$, one of which has a vertical direction we denote by $\mathrm{E}_{0}$ (Fig. 5 shows the intersection locus in red).

It turns out that the set of systems (4.1) with $\alpha=\beta>0$ and having more than six positive solutions can be classified into three families (see [8, Table 6.2 of Chapter 6.5.1]. One of those families of (4.1) turns out to be feasible for our construction; 


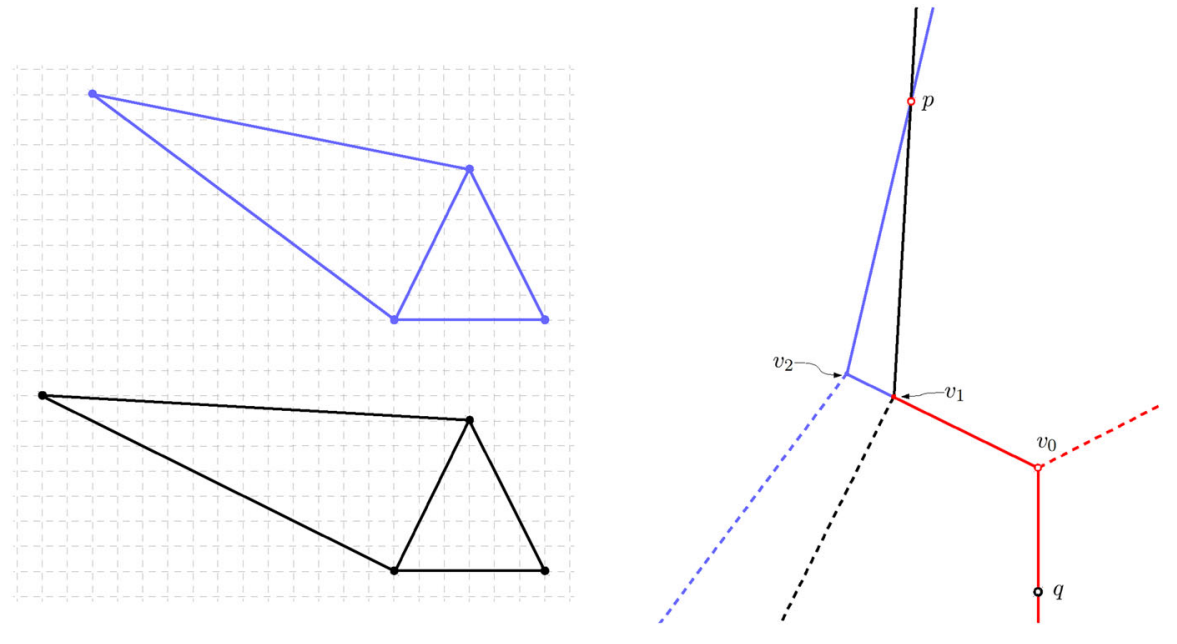

Fig. 5 Newton polytopes and tropical curves associated to a system (4.1) having seven positive solutions. The red lines represent the intersection locus of the tropial curves

it has at most seven positive solutions, and the disposition of their valuations is the following (see Fig. 5).

- The common vertex $v_{0}$ is the valuation of five positive solutions,

- the transversal intersection point $p$ is the valuation of one positive solution, and

- the common vertical edge $\mathrm{E}_{0}$ contains the valuation $q$ of one positive solution.

\subsubsection{Solutions with valuation $v_{0}$}

In order to study the positive solutions to (4.1) with valuation $v_{0}$, we consider the system

$$
\left\{\begin{array}{c}
a_{0}+z_{1}^{m_{1}}+a_{2} z_{1}^{m_{2}} z_{2}^{n_{2}}+a_{3} t^{\alpha} z_{1}^{m_{3}} z_{2}^{n_{3}}=0, \\
c_{0} t^{\gamma_{0}}+b_{4} t^{\beta} z_{1}^{m_{4}} z_{2}^{n_{4}}+c_{2} t^{\gamma_{2}} z_{1}^{m_{2}} z_{2}^{n_{2}}-a_{3} t^{\alpha} z_{1}^{m_{3}} z_{2}^{n_{3}}=0,
\end{array}\right.
$$

with $c_{i} t^{\gamma_{i}}=b_{i}-a_{i}, \operatorname{val}\left(c_{i}\right)=0$ and $\gamma_{i} \geq 0$ for $i=0$, 2. Since the second equation of (4.2) is obtained by substracting the first equation of (4.1) from its second one, the non-degenerate solutions in $\left(\mathbb{K}^{*}\right)^{2}$ (and thus those in $\left(\mathbb{R} \mathbb{K}^{*}\right)^{2}$ ) to (4.1) and of (4.2) coincide. The case-by-case study done in [8, Chapter 6.5$]$ shows that we can hope to obtain a system (4.1) having seven positive solutions if we have

$$
\operatorname{coef}\left(a_{i}\right)=\operatorname{coef}\left(b_{i}\right) \quad \text { for } \quad i=0,2, \quad \text { and } \quad \alpha=\beta=\gamma_{2}<\gamma_{0} \text {. }
$$

In what follows, we assume that (4.2) satisfies the relations in (4.3), and denote by $T_{1}$ and $T_{3}$ the tropical curves corresponding to respectively the first and second polynomials appearing in (4.2). Since the number $\alpha$, appearing in the first equation of (4.2), is positive, the subdivision $\tau_{1}$ of the corresponding Newton polytope $\Delta_{1}$ has the triangle 


$$
\Delta_{1}^{0}:=\left\{\left(\begin{array}{l}
0 \\
0
\end{array}\right),\left(\begin{array}{c}
m_{1} \\
0
\end{array}\right),\left(\begin{array}{c}
m_{2} \\
n_{2}
\end{array}\right)\right\} .
$$

On the other hand, since we have $\alpha=\beta=\gamma_{2}<\gamma_{0}$, the triangulation $\tau_{3}$ of the Newton polytope correspoding to the second equation of (4.2) has the triangle

$$
\Delta_{3}^{0}:=\left\{\left(\begin{array}{c}
m_{2} \\
n_{2}
\end{array}\right),\left(\begin{array}{c}
m_{3} \\
n_{3}
\end{array}\right),\left(\begin{array}{c}
m_{4} \\
n_{4}
\end{array}\right)\right\}
$$

Computing the tropical polynomials of the equations appearing in (4.2) which satisfy (4.3), it is easy to deduce that the vertices $v_{1} \in T_{1}$ and $v_{3} \in T_{3}$ dual to $\Delta_{1}^{0}$ and $\Delta_{3}^{0}$ respectively, coincide at the origin $(0,0)$. Denote by $v_{0}$ the corresponding intersection vertex at the origin in the union $T_{1} \cup T_{3}$.

Consider now the real polynomial system

$$
\left\{\begin{array}{c}
\operatorname{coef}\left(a_{0}\right)+z_{1}^{m_{1}}+\operatorname{coef}\left(a_{2}\right) z_{1}^{m_{2}} z_{2}^{n_{2}}=0 \\
\operatorname{coef}\left(b_{4}\right) z_{1}^{m_{4}} z_{2}^{n_{4}}-\operatorname{coef}\left(a_{3}\right) z_{1}^{m_{3}} z_{2}^{n_{3}}+\operatorname{coef}\left(c_{2}\right) z_{1}^{m_{2}} z_{2}^{n_{2}}=0
\end{array}\right.
$$

which is obtained from (4.2) by multiplying its second equation by $t^{-\alpha}$ and taking $t=0$. Indeed, since the first order terms of all real Puiseux series $a_{i}$ and $b_{j}$ are constant and by assumption, we have $\alpha=\beta=\gamma_{2}<\gamma_{0}$.

Lemma 4.1 If (4.2) has a solution $\left(\lambda_{1}, \lambda_{2}\right) \in\left(\mathbb{K}^{*}\right)^{2}$ such that $\operatorname{Val}\left(\lambda_{1}, \lambda_{2}\right)=v_{0}$, then by taking $t>0$ small enough, the point $\operatorname{Coef}\left(\lambda_{1}, \lambda_{2}\right) \in\left(\mathbb{C}^{*}\right)^{2}$ becomes a solution to the system (4.4).

Proof Recall that $\alpha>0$ and $\alpha=\beta=\gamma_{2}<\gamma_{0}$. Thus the second equation of (4.2) can be re-written as

$$
c_{0} t^{\gamma_{0}-\alpha}+c_{2} z_{1}^{m_{2}} z_{2}^{n_{2}}-a_{3} z_{1}^{m_{3}} z_{2}^{n_{3}}+b_{4} z_{1}^{m_{4}} z_{2}^{n_{4}}=0
$$

with $\gamma_{0}-\alpha>0$. Since $\left(\lambda_{1}, \lambda_{2}\right)$ is a solution to (4.2) satisfying $\operatorname{Val}\left(\lambda_{1}, \lambda_{2}\right)=(0,0)$, the solution $\left(\lambda_{1}, \lambda_{2}\right)$ will converge to $\operatorname{Coef}\left(\lambda_{1}, \lambda_{2}\right)$ when $t>0$ is small enough. Hence, the system (4.2) will converge to (4.4), producing the complex solution $\operatorname{Coef}\left(\lambda_{1}, \lambda_{2}\right)$ to the latter.

The following result gives an explicit description of the solutions in $\left(\mathbb{R} \mathbb{K}_{>0}\right)^{2}$ to (4.2), and thus of those to (4.1), with valuation at $v_{0}$.

Proposition 4.2 Assume that all solutions to (4.2) are non-degenerate. If the system (4.4) has a solution $\left(\rho_{1}, \rho_{2}\right) \in\left(\mathbb{R}_{>0}\right)^{2}$, then (4.2) has a non-degenerate solution $\left(\lambda_{1}, \lambda_{2}\right) \in\left(\mathbb{R} \mathbb{K}_{>0}\right)^{2}$ such that $\operatorname{Val}\left(\lambda_{1}, \lambda_{2}\right)=v_{0}$ and $\operatorname{Coef}\left(\lambda_{1}, \lambda_{2}\right)=\left(\rho_{1}, \rho_{2}\right)$.

Proof Recall that $v_{0}$ is a tropical intersection point of type (III) for the polynomial system (4.2). Denote by $\xi_{1}$ (resp. $\xi_{3}$ ) the 3-valent vertex of the tropical curve associated to the first (resp. second) equation of (4.2) such that $v_{0}=\xi_{1} \cap \xi_{3}$, and denote by $\Delta_{\xi_{1}}$ (resp. $\Delta_{\xi_{3}}$ ) its dual triangle in $\mathbb{R}^{2}$. Note that $\Delta_{\xi_{1}}$ (resp. $\Delta_{\xi_{3}}$ ) is the Newton triangle of 
the first (resp. second) equation of (4.4). E. Brugallé and L. López De Medrano showed in [5, Proposition 3.11] (see also [11, 17, 18] for more details for higher dimension and more exposition relating toric varieties and tropical intersection theory) that the number of solutions of (4.2) with valuation at $v_{0}$ is equal to the mixed volume $\operatorname{MV}\left(\Delta_{\xi_{1}}, \Delta_{\xi_{3}}\right)$ of $\Delta_{\xi_{1}}$ and $\Delta_{\xi_{3}}$ (recall that $\Delta_{v_{0}}=\Delta_{\xi_{1}}+\Delta_{\xi_{3}}$ ). Since we assumed that (4.2) has only non-degenerate solutions in $\left(\mathbb{K}^{*}\right)^{2}$, we get $\operatorname{MV}\left(\Delta_{\xi_{1}}, \Delta_{\xi_{3}}\right)$ distinct solutions of the system (4.2) in $\left(\mathbb{K}^{*}\right)^{2}$ with valuation $v_{0}$. By Lemma 4.1, if $\left(\lambda_{1}, \lambda_{2}\right)$ is a solution to (4.2) and $\operatorname{Val}\left(\lambda_{1}, \lambda_{2}\right)=v_{0}$, then $\operatorname{Coef}\left(\lambda_{1}, \lambda_{2}\right)$ is a solution to (4.4). The number of solutions in $\left(\mathbb{C}^{*}\right)^{2}$ to (4.4) is $\operatorname{MV}\left(\Delta_{\xi_{1}}, \Delta_{\xi_{3}}\right)$. Assuming that the latter system has $\operatorname{MV}\left(\Delta_{\xi_{1}}, \Delta_{\xi_{3}}\right)$ distinct solutions in $\left(\mathbb{C}^{*}\right)^{2}$, we obtain that the map $z:=\left(z_{1}, z_{2}\right) \mapsto \operatorname{Coef}\left(z_{1}, z_{2}\right)$ induces a bijection from the set of solutions to (4.2) in $\left(\mathbb{K}^{*}\right)^{2}$ with valuation at $v_{0}$ onto the set of solutions in $\left(\mathbb{C}^{*}\right)^{2}$ to $(4.4)$.

If $z$ is a solution to (4.2) in $\left(\mathbb{K}^{*}\right)^{2}$ with $\operatorname{Val}(z)=v_{0}$ and $\operatorname{Coef}(z) \in\left(\mathbb{R}^{*}\right)^{2}$, then $z \in\left(\mathbb{R} \mathbb{K}^{*}\right)^{2}$ since otherwise, $z, \bar{z}$ would be two distinct solutions to (4.2) in $\left(\mathbb{K}^{*} \backslash \mathbb{R} \mathbb{K}^{*}\right)^{2}$ such that $\operatorname{Val}(z)=\operatorname{Val}(\bar{z})=v_{0}$ and $\operatorname{Coef}(z)=\operatorname{Coef}(\bar{z})$.

The real system (4.4) has at most five positive solutions. Indeed, since this is a system of two trinomials in two variables (see [13]). The key component of the construction is to find a system (4.4) that has five positive solutions. Once the proper coefficients and exponent vectors for the latter system are obtained, the choice for the complementary coefficients appearing in the system (4.2) becomes easy. Namely, in what follows, we justify the choice of

$$
\left\{\begin{array}{c}
-1+z_{1}^{6}+z_{1}^{3} z_{2}^{6}=0 \\
-\left(\frac{44}{31}\right)^{\frac{5}{6}} \cdot z_{1}^{-12} z_{2}^{9}+z_{1}^{-14} z_{2}^{7}+0.36008 \cdot z_{1}^{3} z_{2}^{6}=0
\end{array}\right.
$$

which belongs to the family of systems (4.4), and has five positive solutions.

\subsubsection{Choosing the coefficients}

First, the coefficients coef $\left(a_{0}\right)$ and coef $\left(a_{2}\right)$ cannot be both positive, since otherwise the first equation of (4.4) would not have positive solutions. Among the three other possibilities, we make the arbitrary choice coef $\left(a_{0}\right)<0$ and coef $\left(a_{2}\right)>0$. Now, dividing the first equation by $-\operatorname{coef}\left(a_{0}\right)$, and doing a change of coordinates of type $\left(z_{1}, z_{2}\right) \mapsto\left(a \cdot z_{1}, b \cdot z_{2}\right)$ for some $(a, b) \in\left(\mathbb{R}_{>0}\right)^{2}$, we assume that $\operatorname{coef}\left(a_{2}\right)=-\operatorname{coef}\left(a_{0}\right)=1$. Having done that, we are still able to control only one coefficient of (4.4). Similarly, since we can divide the second equation by any real number without changing the number of non-degenerate positive solutions, for simplicity we set coef $\left(a_{3}\right)=-1$. The system (4.4) can now be written as

$$
\left\{\begin{array}{c}
-1+z_{1}^{m_{1}}+z_{1}^{m_{2}} z_{2}^{n_{2}}=0 \\
\operatorname{coef}\left(b_{4}\right) z_{1}^{m_{4}} z_{2}^{n_{4}}+z_{1}^{m_{3}} z_{2}^{n_{3}}+\operatorname{coef}\left(c_{2}\right) z_{1}^{m_{2}} z_{2}^{n_{2}}=0 .
\end{array}\right.
$$

As for the remaining two coefficients of (4.6), we have the following result. 
Proposition 4.3 If we have

$$
\begin{aligned}
& \operatorname{coef}\left(c_{2}\right)=-0.36008, \quad \operatorname{coef}\left(b_{4}\right)=-\left(\frac{44}{31}\right)^{\frac{5}{6}} \\
& \frac{m_{3} \cdot n_{2}-m_{2} \cdot n_{3}}{m_{1} \cdot n_{2}}=-\frac{35}{12}, \frac{m_{4} \cdot n_{2}-m_{2} \cdot n_{4}}{m_{1} \cdot n_{2}}=-\frac{11}{4}, \text { and } \frac{n_{4}-n_{3}}{n_{2}}=\frac{1}{3},
\end{aligned}
$$

then the system (4.6) has five non-degenerate positive solutions.

Proof First, we define a univariate function $f$ such that for some constant $c$, the equation $f=c$ has the same number of solutions in ]0, 1[ as that of positive solutions to (4.6). We write the first equation of (4.6) as $z_{2}=x^{k}(1-x)^{l}$, where $x:=z_{1}^{m_{1}}$, $k=-m_{2} /\left(m_{1} n_{2}\right)$ and $l=1 / n_{2}$. It is clear that $\left.z_{1}, z_{2}>0 \Leftrightarrow x \in I_{0}:=\right] 0,1[$.

Since we are looking for solutions to (4.6) with non-zero coordinates, we can divide its second equation by $z_{1}^{m_{2}} z_{2}^{n_{2}}$. Plugging $z_{1}$ and $z_{2}$ in the second equation of (4.6), we get

$$
\operatorname{coef}\left(c_{2}\right)+x^{k_{3}}(1-x)^{l_{3}}+\operatorname{coef}\left(b_{4}\right) x^{k_{4}}(1-x)^{l_{4}}=0
$$

where $k_{i}=\frac{m_{i} n_{2}-m_{2} n_{i}}{m_{1} n_{2}}$ and $l_{i}=\frac{n_{i}-n_{2}}{n_{2}}$ for $i=3,4$.

The number of positive solutions to (4.6) is equal to the number of solutions to (4.8) in $I_{0}$. Therefore we want to compute values of $\operatorname{coef}\left(c_{2}\right), \operatorname{coef}\left(b_{4}\right)$ and $\left(m_{i}, n_{i}\right)$ for $i=1,2,3,4$ such that $f(x)+\operatorname{coef}\left(c_{2}\right)=0$ has five solutions in $I_{0}$, where

$$
f(x):=x^{k_{3}}(1-x)^{l_{3}}+\operatorname{coef}\left(b_{4}\right) \cdot x^{k_{4}}(1-x)^{l_{4}} .
$$

Note that the function $f$ has no poles in $I_{0}$, thus by Rolle's theorem we have $\sharp\{x \in$ $\left.I_{0} \mid f(x)=-\operatorname{coef}\left(c_{2}\right)\right\} \leq \sharp\left\{x \in I_{0} \mid f^{\prime}(x)=0\right\}+1$. The derivative $f^{\prime}$ is expressed as

$$
x^{k_{3}-1}(1-x)^{l_{3}-1} \rho_{3}(x)+\operatorname{coef}\left(b_{4}\right) \cdot x^{k_{4}-1}(1-x)^{l_{4}-1} \rho_{4}(x),
$$

where $\rho_{i}(x)=k_{i}-\left(k_{i}+l_{i}\right) \cdot x$ for $i=3$, 4. For $x \in$ ]0, 1[, we have $f^{\prime}(x)=0 \Leftrightarrow$ $\phi(x)=1$, where

$$
\phi(x):=-\operatorname{coef}\left(b_{4}\right) \frac{x^{k_{4}-k_{3}}(1-x)^{l_{4}-l_{3}} \rho_{4}(x)}{\rho_{3}(x)} .
$$

From the discussion above, since (4.6) has at most five positive solutions, the equation (4.10) has at most four positive solutions.

Consider now the system

$$
x^{6}+(44 / 31) y^{3}-y=y^{6}+(44 / 31) x^{3}-x=0,
$$

taken from [7], which has five positive solutions. Making similar operations to it produces the rational function 


$$
\phi_{0}(x)=(44 / 31)^{5 / 6} \cdot \frac{x^{1 / 6}(1-x)^{1 / 3} \cdot(-11 / 4+9 x / 4)}{(-35 / 12+11 x / 4)},
$$

such that $\phi_{0}(x)=1$ has four positive solutions in the interval $I_{0}$. Thus, if the relevant equalities appearing in (4.7) are satisfied, the equation $\phi(x)=1$, with $\phi$ appearing in (4.10), has four positive solutions in $I_{0}$.

Assuming that this is the case, it is only left to compute coef $\left(c_{2}\right)$. Plotting the function $f: \mathbb{R} \rightarrow \mathbb{R}, x \mapsto f(x)$, we get that the graph of $f$ has four critical points contained in $I_{0}$ with critical values situated above the $x$-axis. Moreover, this graph intersects transversally the line $\{y=0.36008\}$ in five points with the first coordinates belonging to $I_{0}$. Taking coef $\left(c_{2}\right)=-0.36008$, the equation $f(x)=0.36008$ has five non-degenerate positive solutions in $I_{0}$, and we have finished the proof.

\subsubsection{Choosing the exponent vectors}

In what follows, we show how to find $\left(m_{i}, n_{i}\right) \in \mathbb{Z}^{2}$ for $i=1,2,3,4$, satisfying the equalities in (4.7) so that (4.6) has five non-degenerate positive solutions, and (4.2) has seven.

Recall that $T_{1}$ and $T_{2}$ denote the tropical curves associated respectively to the first and second equations of the original system (4.1). From the discussion in [8, Chapter 6.5.1.1], imposing $\alpha=\beta=\gamma_{2}<\gamma_{0}$ makes it impossible for the vertical edge $\mathrm{E}_{0}$ of the intersection locus $T_{1} \cap T_{2}$ to contain the valuation of more than one positive solution to (4.1) (see [8, Chapters 6.2 and 6.4.1] for the exposition on the method used to approximate the positive solutions to (4.1), where the tropical curves intersect at components of type (I)).

Assume in what follows that $\mathrm{E}_{0}$ contains the valuation of one positive solution to (4.1). This implies that both $n_{3}$ and $n_{4}$ are positive (see [8, Chapter 6.5.2]), and thus all the vertices of both Newton polytopes of the polynomials appearing in (4.1) have non-negative second coordinate (recall that $n_{2}$ is assumed to be positive). Since in addition we have $\alpha>0$, the edge $\mathrm{E}_{0}$ in the tropical picture of $T_{1} \cap T_{2}$, is a half-ray with direction $(0,-1)$ (see Fig. 5 for an example).

From the discussion in [8, Chapter 6.5.1], if (4.1) has more than six positive solutions, then $v_{0}$ is the only non-transversal intersection point of type (III). Namely, an extra intersection point $v$ of type (III) imposes additional restrictions on the support of (4.1) (and consequently, on the support of (4.4)). These restrictions force $v$ to be the valuation of at most one positive solution, they additionally prohibit both (4.4) having five positive solutions, and $T_{1} \cap T_{2}$ having transversal intersections.

Recall that from the beginning of Sect. 4.1, we are assuming $m_{1}$ and $n_{2}$ to be positive, and suppose furthermore in what follows that $m_{2}$ is also positive. The equalities in (4.7) show that $l_{i}>0, k_{i}<0$ and $k_{i}<l_{i}$ for $i=3,4$, therefore we have $0<n_{2}<n_{i}$, $m_{i} n_{2}-n_{i} m_{2}<0$ and $\left(m_{i}-m_{1}\right) n_{2}-n_{i}\left(m_{2}-m_{1}\right)<0$ for $i=3$, 4. Plotting the three points $(0,0),\left(m_{1}, 0\right)$ and $\left(m_{2}, n_{2}\right)$, we deduce from the latter inequalities that the points $\left(m_{3}, n_{3}\right)$ and $\left(m_{4}, n_{4}\right)$ belong to the region $B_{1}$ of Fig. 6.

We also deduce from equalities in (4.7) that $l_{4}>l_{3}$ and $k_{4}>k_{3}$, and thus $n_{4}>n_{3}$ and $\left(m_{4}-m_{3}\right) n_{2}-\left(n_{4}-n_{3}\right) m_{2}>0$. Fixing $\left(m_{3}, n_{3}\right)$ in the region $B_{1}$, we obtain that $\left(m_{4}, n_{4}\right)$ belongs to the triangle $B_{1,1}$ depicted in Fig. 6. 

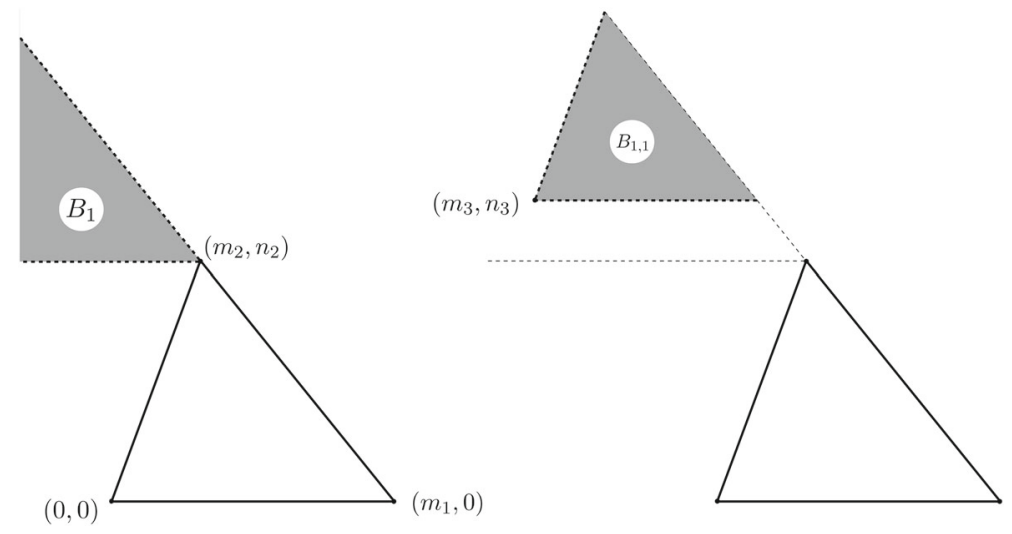

Fig. 6 The region $B_{1}$ and triangle $B_{1,1}$

Note that the vertex $v_{1}$ (resp. $v_{2}$ ) of $T_{1}$ (resp. $T_{2}$ ) has coordinates

$$
\frac{\alpha}{m_{3} n_{2}-n_{3} m_{2}}\left(n_{2},-m_{2}\right) \quad\left(\operatorname{resp} . \frac{\alpha}{m_{4} n_{2}-n_{4} m_{2}}\left(n_{2},-m_{2}\right)\right),
$$

and thus from $m_{3} n_{2}-n_{3} m_{2}<m_{4} n_{2}-n_{4} m_{2}<0$, we deduce that the first coordinate of $v_{2}$ is smaller than that of $v_{1}$ (see Fig. 5).

All these restrictions impose that there exists a transversal intersection point of $T_{1}$ and $T_{2}$. Moreover, since coef $\left(b_{4}\right)<0$ [see (4.7)], coef $\left(a_{3}\right)=-1$ [from (4.6)] and $\operatorname{coef}\left(a_{0}\right)=\operatorname{coef}\left(b_{0}\right)=-1$, the intersection point $p$ is the valuation of a positive solution to (4.1). This follows from [8, Proposition 6.27].

The constant coef $\left(c_{0}\right)$ should be a positive number so that (4.1) would have a positive solution with valuation in $\mathrm{E}_{0}$. This constant can take any positive value, and for computational reasons we choose it to be 0.36008 .

According to this analysis, a valid choice of exponents and coefficients of (4.1) is $m_{1}=6,\left(m_{2}, n_{2}\right)=(3,6),\left(m_{3}, n_{3}\right)=(-14,7),\left(m_{4}, n_{4}\right)=(-12,9), a_{0}=-1$, $a_{2}=1, a_{3}=-1, b_{0}=-1+0.36008 \cdot t^{\gamma_{0}}, b_{2}=1-0.36008 \cdot t^{\alpha}$ (verifying $\left.\gamma_{0}>\alpha>0\right)$, and $b_{4}=-(44 / 31)^{5 / 6}$. Therefore, the system

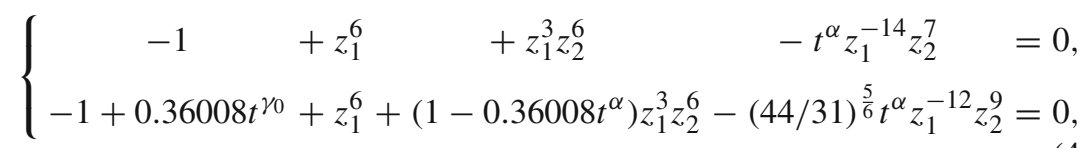

which has seven non-degenerate positive solutions, proves Theorem 1.1.

\subsubsection{A software computation}

Using Maple 17 as well as the libraries FGb and RS, Pierre-Jean Spaenlehauer [21] provided us with a computation he made of the non-degenerate positive solutions to a system (4.12) for $\gamma_{0}=7$ and $\alpha=1$ that goes as follows. For computational reasons, 
he has replaced the real number $(44 / 31)^{5 / 6}$ in (4.12) by the fraction

$$
\frac{26807502408507435267952730104920543812845885439976}{20022295568917288472920446333489413342983920443429}
$$

which approximates $(44 / 31)^{5 / 6}$. For $t=1 / 100000$, the software has found seven positive solutions. An approximation of these solutions goes as follows.

$$
\begin{gathered}
(0.99999,0.00001),(0.99171,0.60681),(0.96651,0.76771),(0.95765,0.79907), \\
(0.95201,0.81642),(0.88602,0.95151),(0.53645,1.61099) .
\end{gathered}
$$

Acknowledgements Open access funding provided by Max Planck Society. I am very grateful to Frédéric Bihan for fruitful discussions and guidance. I would like to thank Pierre-Jean Spaenlehauer for computations that approximated the positive solutions to the system that was constructed to prove Theorem 1.1. I also thank the anonymous referee for helpful remarks and suggestions on earlier versions of this paper.

Open Access This article is distributed under the terms of the Creative Commons Attribution 4.0 International License (http://creativecommons.org/licenses/by/4.0/), which permits unrestricted use, distribution, and reproduction in any medium, provided you give appropriate credit to the original author(s) and the source, provide a link to the Creative Commons license, and indicate if changes were made.

\section{References}

1. Bertrand, B., Bihan, F.: Intersection multiplicity numbers between tropical hypersurfaces. In: Algebraic and Combinatorial Aspects of Tropical Geometry. Contemporary Mathematics, vol. 589, pp. 1-19. American Mathematical Society, Providence (2013)

2. Bihan, F.: Polynomial systems supported on circuits and dessins d'enfants. J. Lond. Math. Soc. (2) 75(1), 116-132 (2007)

3. Bihan, F.: Irrational mixed decomposition and sharp fewnomial bounds for tropical polynomial systems. arXiv preprint arXiv:1410.7905 (to appear in Discrete and Computational Geometry) (2014)

4. Brugallé, E., Itenberg, I., Mikhalkin, G., Shaw, K.: Brief introduction to tropical geometry. In: Proceedings of the Gökova Geometry-Topology Conference 2014, pp. 1-75. Gökova Geometry/Topology Conference (GGT), Gökova (2015)

5. Brugallé, E.A., de Medrano, L.M.L.: Inflection points of real and tropical plane curves. J. Singul. 4, 74-103 (2012)

6. Bihan, F., Sottile, F.: New fewnomial upper bounds from Gale dual polynomial systems. Mosc. Math. J. 7(3), 387-407 (2007). 573

7. Dickenstein, A., Jean-Maurice, R., Rusek, K., Shih, J.: Extremal real algebraic geometry and $\mathcal{A}$ discriminants. Mosc. Math. J. 7(3), 425-452 (2007). 574

8. El Hilany, B.: Tropical geometry and polynomial systems. Ph.D. thesis, Comunauté Université Grenoble Alpes (2016). https://www.math.uni-tuebingen.de/user/boel/Thesis.pdf

9. Haas, B.: A simple counterexample to Kouchnirenko's conjecture. Beitr. Algebra Geom. 43(1), 1-8 (2002)

10. Einsiedler M, Kapranov M, Lind D (2007) Non-archimedean amoebas and tropical varieties. Journal für die reine und angewandte Mathematik 2006(601):139-157. https://doi.org/10.1515/CRELLE.2006. 097

11. Katz, E.: A tropical toolkit. Expo. Math. 27(1), 1-36 (2009)

12. Khovanskiı̌, A.G.: Fewnomials. Translations of Mathematical Monographs, vol. 88. American Mathematical Society, Providence (1991). Translated from the Russian by S. Zdravkovska

13. Li, T.-Y., Rojas, J.-M., Wang, X.: Counting real connected components of trinomial curve intersections and $m$-nomial hypersurfaces. Discrete Comput. Geom. 30(3), 379-414 (2003)

14. Mikhalkin, G.: Tropical geometry and its applications. In: International Congress of Mathematicians, vol. II, pp. 827-852. European Mathematical Society, Zürich (2006) 
15. Mikhalkin, G., Rau, J.: Tropical geometry (in preparation)

16. Maclagan, D., Sturmfels, B.: Introduction to Tropical Geometry, vol. 161. American Mathematical Society, Providence (2015)

17. Osserman, B., Payne, S.: Lifting tropical intersections. Doc. Math. 18, 121-175 (2013)

18. Rabinoff, J.: Tropical analytic geometry, newton polygons, and tropical intersections. Adv. Math. 229(6), 3192-3255 (2012)

19. Renaudineau, A.: Constructions de surfaces algébriques réelles. Ph.D. thesis, Paris 6 (2015)

20. Sottile, F.: Real solutions to equations from geometry. University Lecture Series, vol. 57. American Mathematical Society, Providence (2011)

21. Spaenlehauer P-J (2016) A software computation of a polynomial system having many positive solutions. Pers commun

22. Sturmfels, B.: Viro's theorem for complete intersections. Ann. Scuola Norm. Super. Pisa Cl. Sci. (4) 21(3), 377-386 (1994)

23. Viro, O.Y.: Gluing of plane real algebraic curves and constructions of curves of degrees 6 and 7. In: Topology (Leningrad, 1982). Lecture Notes in Mathematics, vol. 1060, pp. 187-200. Springer, Berlin (1984)

24. Viro, O.Y.: Real plane algebraic curves: constructions with controlled topology. Algebra Anal. 1(5), $1-73$ (1989)

25. Viro, O.: Dequantization of Real Algebraic Geometry on Logarithmic Paper, pp. 135-146. Birkhäuser Basel, Basel (2001) 